Hautarzt 2017 $\cdot 68: 43-49$

DOI 10.1007/s00105-016-3905-0

Online publiziert: 15. Dezember 2016

๑) Springer Medizin Verlag Berlin 2016

CrossMark

\author{
P. Nenoff $\cdot$ A. Manos ${ }^{2} \cdot$ I. Ehrhard ${ }^{3} \cdot$ C. Krüger $\cdot$ U. Paasch ${ }^{4} \cdot$ P. Helmbold ${ }^{5}$.

\section{W. Handrick} \\ ${ }^{1}$ Labor für medizinische Mikrobiologie, Partnerschaft Prof. Dr. med. Pietro Nenoff \& Dr. med. Constanze \\ Krüger, Rötha/OT Mölbis, Deutschland \\ ${ }^{2}$ Beratungsstelle für sexuell übertragbare Krankheiten und AIDS, Gesundheitsamt Leipzig, Leipzig, \\ Deutschland \\ ${ }^{3}$ Abt. Medizinische Mikrobiologie und Hygiene, Landesuntersuchungsanstalt für das Gesundheits- und \\ Veterinärwesen Sachsen, Dresden, Deutschland \\ ${ }^{4}$ Klinik und Poliklinik für Dermatologie, Venerologie und Allergologie, Universität Leipzig, Leipzig, \\ Deutschland \\ ${ }^{5}$ Hautklinik des Klinikums der Universität Heidelberg, Heidelberg, Deutschland \\ ${ }^{6}$ Institut für Medizinische Diagnostik Oderland, Frankfurt (Oder), Deutschland
}

\title{
Nichtvirale sexuell übertragene Infektionen - Epidemiologie, Klinik, Labordiagnostik und Therapie
}

\section{Teil 1: Gonokokken}

\section{Ätiologie und Pathogenese}

Die Gonorrhö als nahezu ausschließlich sexuell übertragene Erkrankung betrifft vornehmlich die Schleimhaut des Urogenitaltraktes von Mann und Frau. Es sind jedoch auch extragenitale Lokalisationen (Anorektalbereich, pharyngeal und okulär) zu bedenken [23]. Bei 80-90\% der Patienten bleibt sie auf den Bereich der Eintrittspforten beschränkt. Es kann aber auch zu aufsteigenden Infektionen mit entzündlicher Verklebung sowie Vernarbung von Nebenhoden, Samenstrang und Tuben mit nachfolgender Sterilität kommen.

\section{Epidemiologie}

\section{Gonorrhö-Inzidenz in den USA}

Die WHO schätzt, dass weltweit jährlich 106 Mio. Neuinfektionen durch Neisseria (N.) gonorrhoeae auftreten [24]. Allein in den USA erkranken pro Jahr etwa 800.000 Menschen [5]. In den USA

Herrn Prof. Dr. med. Uwe-Frithjof Haustein, Leipzig, zum 80. Geburtstag gewidmet. wurde im Jahr 2015 die bislang höchste Inzidenzrate für die Gonorrhö ermittelt. Diese lag bei 124 pro 100.000 der Bevölkerung, das ist ein Anstieg um 13\% im Vergleich zu 2014. Die Gonorrhö war am häufigsten unter Afroamerikanern und Indianern sowie den Ureinwohnern Alaskas [6]. Betroffen sind in den USA junge homosexuelle sowie bisexuelle Menschen. Der Hauptteil der Infektionen betrifft Mädchen und Frauen im Alter von 15 bis 24 Jahren und Männer von 20 bis 24 Jahren. Die neuen STI („sexually transmitted infection“)Zahlen in den USA hängen aus Sicht von Prof. Brockmeyer, dem Präsidenten der DSTIG (Deutsche STI-Gesellschaft) mit der Reduzierung der Versorgungsstrukturen in den USA zusammen. Die vorhandenen Versorgungsstrukturen erreichen zudem nur unzureichend den Lebenskontext der Nativ- und Afroamerikaner sowie der Menschen mit lateinamerikanischer Abstammung.

\section{Epidemiologie der Gonorrhö in Europa, Deutschland und Sachsen}

Für Deutschland liegen aufgrund fehlender Meldepflicht keine aktuellen Daten zur Epidemiologie vor. In Sachsen stehen jedoch aufgrund der hier geltenden erweiterten Meldeverordnung entsprechend dem Infektionsschutzgesetz (IfSG) die aktuellen Meldedaten zur GonorrhöInzidenz zur Verfügung.

Vor 2001 war die Gonorrhö in Deutschland meldepflichtig. Das seit 2001 geltende IfSG sieht keine Meldepflicht mehr vor. Entsprechend IfSG (\$ 7 Abs. 3) sind heute Syphilis, HIVNachweis und auch (sexuell übertragbare) Virushepatitiden dem Robert KochInstitut (RKI) zu melden. HIV, Syphilis und Virushepatitiden werden direkt vom Labor dem RKI gemeldet. In Sachsen gilt die erweiterte Meldeverordnung laut IfSG. Neben der Syphilis sind in Sachsen N.-gonorrhoeae-, Chlamydia trachomatis- und auch genitale Mykoplasmennachweise meldepflichtig (Labormeldepflicht, $\$ 2$ Abs. 1 Nr. 13, $\S 2$ Abs. 2, $\$ 3$ Abs. 3) [32]. Seit 2009 kam es zu einer eindrucksvollen Zunah- 


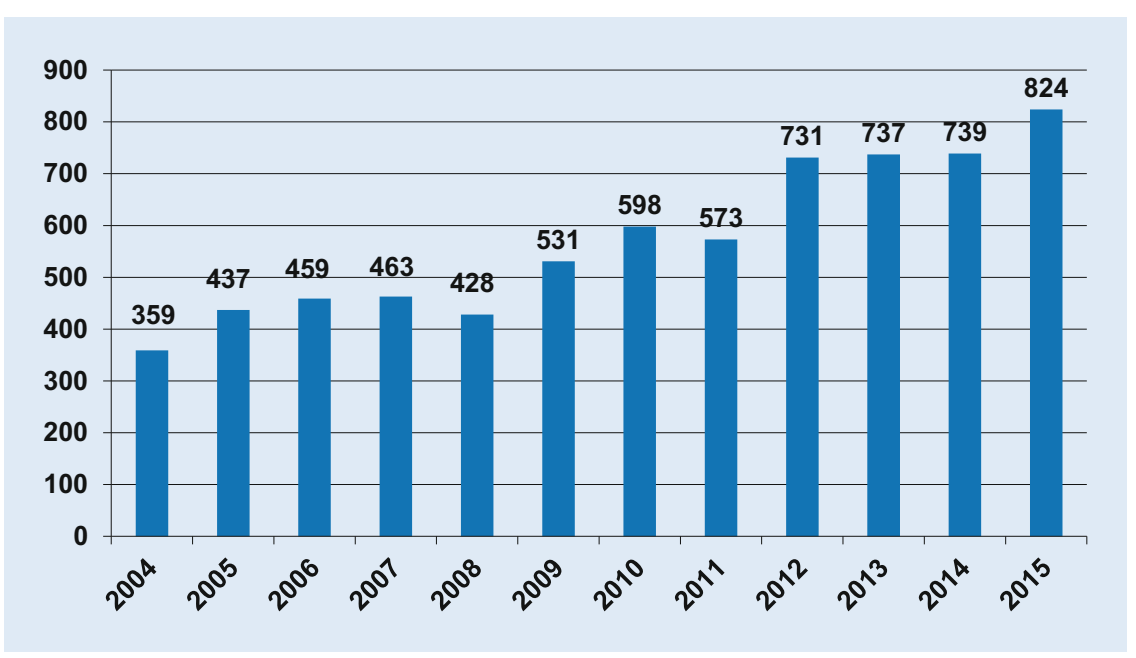

Abb. 1 A Anzahl der in Sachsen gemeldeten genitalen Neisseria-gonorrhoeae-Infektionen

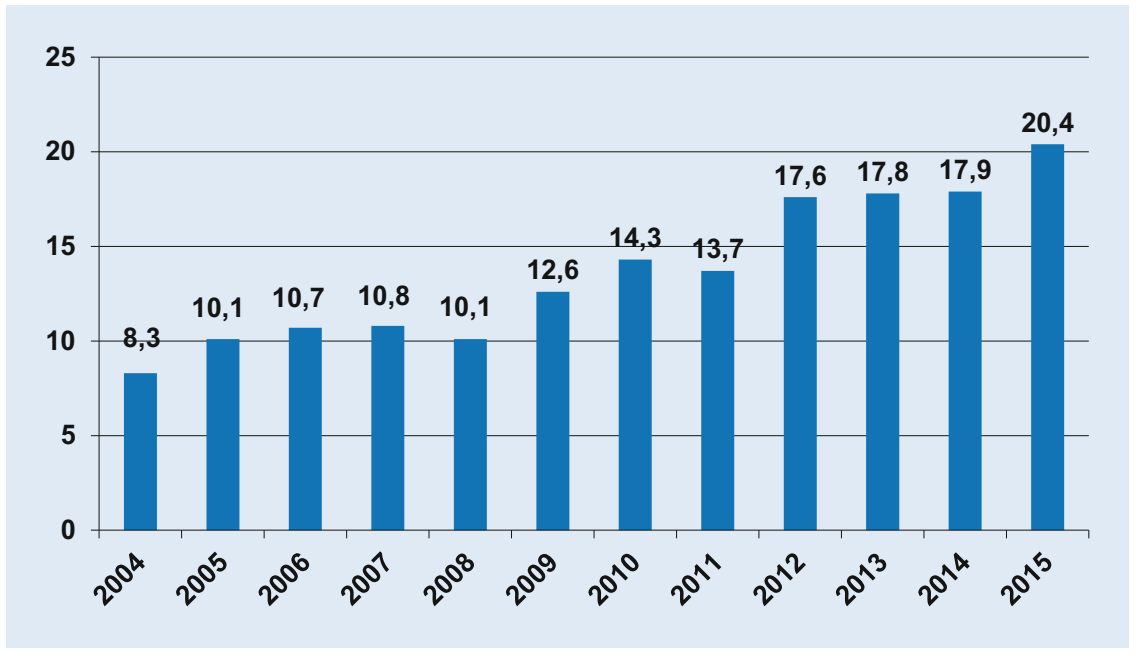

Abb. 2 A Neisseria-gonorrhoeae-Inzidenzen von 2004 bis 2015 in Sachsen

me der gemeldeten Infektionen durch N. gonorrhoeae (• Abb. 1). Im Jahr 2014 wurden 739 Infektionen gemeldet; 824 gemeldete N.-gonorrhoeae-Infektionen im Jahr 2015 sprechen für einen weiteren Anstieg der Gonorrhö-Inzidenz in Sachsen. Über eine adäquate Zunahme der Infektionszahlen in Deutschland kann nur spekuliert werden.

\section{》) Seit 2009 kam es in Sachsen zu einer deutlichen Zunahme der gemeldeten Infektionen durch N. gonorrhoeae}

Im Jahr 2015 betrug die Inzidenz der gemeldeten Gonorrhö-Fälle in Sachsen
Der Vergleich der sächsischen Großstädte ergibt, dass der Stadtkreis Leipzig 2015 mit 233 gemeldeten Erkrankungen die höchste Inzidenz (43,8/100.000 Einwohner) aufwies. Es folgten Dresden (157, Inzidenz 29,6) und Chemnitz (101, Inzidenz 41,7). Ursachen für die hohen Zahlen für Leipzig - wie eine häufigere Labordiagnostik seitens der Ärzte, ein ausgesprochen gutes Meldeverhalten der dortigen Laborpraxen, aber auch ein risikobehaftetes Sexualverhalten - können nur vermutet werden.

\section{Klinisches Bild}

Symptome einer gonorrhoischen Urethritis des Mannes sind Prickeln in der Harnröhre, Brennen beim Wasserlassen und eitriger, gelbgrüner Fluor, bestehend aus Gonokokken und Leukozyten [13]. Unbehandelt wird der Fluor nach einigen Wochen glasig-schleimig und tritt nur noch morgens als Gonokokkenhaltiger Tropfen in Erscheinung („Bonjour-Tropfen"). Die Urethritis durch $N$. gonorrhoeae ist dagegen bei Mädchen und Frauen häufiger als beim Mann asymptomatisch. Bei der gonorrhoischen Vaginitis tritt grünlich-eitriger bzw. schleimiger Fluor auf.

Zervizitis und entzündliche Beckenerkrankungen („pelvic inflammatory disease", PID) sind gefürchtete Komplikationen. $\mathrm{Zu}$ nennen sind hier auch Bauchhöhlenschwangerschaft, Infertilität und chronische Beckenschmerzen. Bei Männern kann die Gonorrhö als symptomatische Urethritis und Epididymitis imponieren [25]. Sowohl Chlamydienals auch N.-gonorrhoeae-Infektionen begünstigen die HIV-Übertragung, was vorzugsweise in Regionen mit hoher Prävalenz, beispielsweise in Afrika südlich der Sahara Bedeutung hat.

\section{Pharyngeale Gonorrhö}

Die pharyngeale Gonorrhö tritt nach orogenitalen Kontakten auf. Symptome finden sich jedoch nicht auf dem pharyngealen Plattenepithel, sodass man auf anamnestische Hinweise des Patienten angewiesen ist bzw. auf das Ergebnis des mikrobiologischen Abstrichs. (Zur Bewertung der kulturellen und molekularbiolo- 
gischen Diagnostik mittels Nukleinsäureamplifikationstechniken [NAT] s. im Abschn. Diagnostik.)

\section{Rektale Gonorrhö}

Auch die rektale Gonorrhö verläuft oft asymptomatisch. Bei Frauen kommt es zur rektalen Gonorrhö infolge einer Schmierinfektion vom Fluor vaginalis ausgehend oder nach Analverkehr. Eine Proktitis bei homosexuellen Männern sollte Anlass sein, auch an eine Gonorrhö zu denken und eine entsprechende Diagnostik durchzuführen. Die Symptome sind Schmerzen, Tenesmen und auch Fluor, ein asymptomatischer Verlauf ist jedoch ebenfalls möglich.

Aktuell wurde nachgewiesen, dass Speichel als Gleitmittel beim Analverkehr von Männern, die Sex mit Männern haben (MSM), signifikant mit rektaler Gonorrhö assoziiert ist [7].

\section{Disseminierte Gonokokken- Infektion}

$\mathrm{Zu}$ einer Disseminierung kommt es bei bis zu $3 \%$ der Patienten nach einer lokalen Gonorrhö. Zum üblichen klinischen Bild der disseminierten GonokokkenInfektion gehören Hautausschlag (auch vaskulitische Hauterscheinungen), Polyarthralgie und Tenosynovitis [20]. Dazu können Fieberschübe kommen. Kürzlich wurde eine disseminierte GonokokkenInfektion bei einer 28-jährigen immunsupprimierten Patientin beschrieben, die wegen einer paroxysmalen nächtlichen Hämoglobinurie eine Behandlung mit Eculizumab erhielt [16]. Die Gonokokken wurden mittels Blutkultur nachgewiesen.

\section{Diagnostik}

\section{Materialentnahme bei Verdacht auf genitale Gonorrhö}

Abstriche für den mikroskopischen und kulturellen Gonokokken-Nachweis sind prinzipiell von allen potenziellen Infektionslokalisationen möglich. Bei der Beurteilung ist jedoch zu bedenken, dass es auch apathogene Neisserien-Arten gibt, die insbesondere bei Pharyngealabstri-

Hautarzt 2017 -68:43-49 DOI 10.1007/s00105-016-3905-0

(c) Springer Medizin Verlag Berlin 2016

P. Nenoff · A. Manos · I. Ehrhard · C. Krüger · U. Paasch · P. Helmbold · W. Handrick

Nichtvirale sexuell übertragene Infektionen - Epidemiologie, Klinik, Labordiagnostik und Therapie. Teil 1: Gonokokken

\section{Zusammenfassung}

Die Weltgesundheitsorganisation (WHO)

schätzt, dass weltweit pro Tag 1 Mio.

Menschen mit einer STI (,sexually transmitted infection") infiziert werden. Die Gonorrhö wird nahezu ausschließlich sexuell übertragen und betrifft vornehmlich die Schleimhaut des Urogenitaltraktes, möglich sind jedoch auch extragenitale Lokalisationen (Anorektalbereich). Laut Infektionsschutzgesetz (IfSG) sind heute in Deutschland nur Syphilis und HIV-Nachweis zu melden. In Sachsen gilt die erweiterte Meldeverordnung laut IfSG, sodass neben der Syphilis auch Neisseria-gonorrhoeae-, Chlamydia trachomatis- und genitale Mykoplasmennachweise meldepflichtig sind. Seit 2009 kam es in Sachsen zu einer eindrucksvollen Zunahme der gemeldeten Infektionen durch Neisseria gonorrhoeae, im Jahr 2015 waren es 824 Meldungen.
Besorgniserregend ist die Zunahme der Resistenz von Neisseria gonorrhoeae gegenüber Penicillin, Doxycyclin, Ciprofloxacin und neuerdings auch gegenüber Azithromycin und Drittgenerationscephalosporinen. Der ursprünglich in Japan aufgetretene sog. Superbug („Superbazillus“) von Neisseria gonorrhoeae mit Multiresistenz gegenüber den meisten verfügbaren oralen Antibiotika ist mittlerweile auch in Europa angekommen. Die Standardtherapie der Gonorrhö erfolgt heute mit Ceftriaxon (i. v. oder i. m.) zusammen mit Azithromycin p. o., beides als Einmalgabe.

\section{Schlüsselwörter}

Neisseria gonorrhoeae · Resistenz - Ceftriaxon · Cefixim · Azithromycin

\section{Non-viral sexually transmitted infections - Epidemiology, clinical manifestations, diagnostics and therapy. Part 1: Gonococci}

\begin{abstract}
Approximately 1 million people are infected per day worldwide by one or more sexually transmitted infections (STI) as estimated by the World Health Organization (WHO). Gonorrhoea represents an almost exclusively sexually transmitted infection, which predominantly affects mucous membranes of the genitourinary tract. Extragenital localization of infections is also possible, e. $g$. in the anorectal region. Currently, only syphilis and human immunodeficiency virus (HIV) are notifiable diseases according to the Infection Protection Act in Germany. In Saxony, an extended registration ordinance according to the German Infection Protection Act is in force, which means that besides syphilis the laboratory detection of Neisseria gonorrhoeae, Chlamydia trachomatis and genital mycoplasms are also notifiable infections. In particular, beginning in 2009 in Saxony
\end{abstract}

\begin{abstract}
a spectacular increase of registered infections due to $N$. gonorrhoeae was observed and in 2015 altogether 824 infections due to $N$. gonorrhoeae were reported. Alarming is the increase in resistance of $N$. gonorrhoeae against penicillin, doxycycline, ciprofloxacin and recently also against azithromycin and third generation cephalosporins. The socalled superbug of N. gonorrhoeae, which originated in Japan with multidrug resistance against most of the currently available oral antibiotics, has now arrived in Europe. Intramuscular or intravenous injection of ceftriaxone plus oral azithromycin, each given as single dose is the standard therapy for gonorrhoea.
\end{abstract}

\section{Keywords}

Neisseria gonorrhoeae - Resistance .

Ceftriaxone $\cdot$ Cefixime $\cdot$ Azithromycin chen zu falsch positiven Ergebnissen im Grampräparat führen können. NAT werden heute aufgrund der höheren Empfindlichkeit und Spezifität bevorzugt eingesetzt. Die DNA-basierten Methoden sind von der U.S. Food and Drug Administration(FDA) für die Untersuchung von urogenitalen Proben zugelassen. Da$\mathrm{zu}$ zählen endozervikale und vaginale Abstriche sowie urethrale Abstriche vom Mann, jedoch auch Urin von der Frau und vom Mann. Bei Verwendung von Urin ist den Patienten unbedingt zu erklären, dass nur der Erststrahlurin zu verwen- 


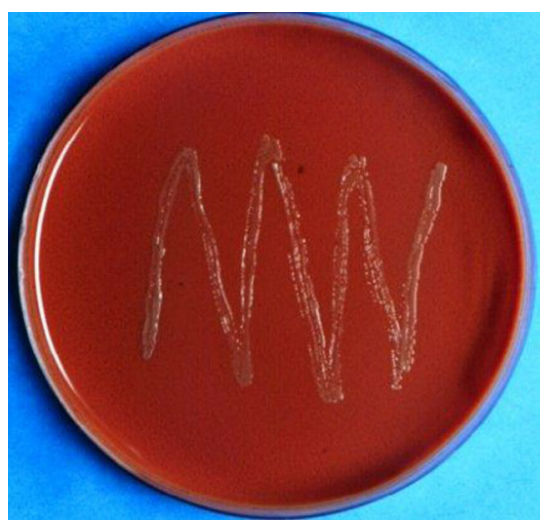

Abb. 3 ॥ Neisseria gonorrhoeae: kleine, glatte, pigmentlose, grau-opake Kolonien von 0,6-1,5 mm Durchmesser nach 2 Tagen Wachstum bei $36^{\circ} \mathrm{C}$ unter mikroaerophilen Bedingungen auf Selektivmedium nach Thayer-Martin (,,Schokoladenagar")

den ist, nicht der Mittelstrahlurin, der bei Harnweginfektionen untersucht wird. Für Rektal- und Pharyngealabstriche gilt diese Zulassung der FDA nicht, im Labor werden die Methoden dafür trotzdem unter Vorbehalt eingesetzt. Manche NAT sind zudem für die Eigenentnahme von Vaginalabstrichen zugelassen.

Vaginalabstriche haben sich im Vergleich mit endozervikalen Abstrichen oder Erststrahlurin als mindestens genauso empfindlich zum molekularbiologischen N.-gonorrhoeae-Nachweis erwiesen. Für das Cobas $4800 \mathrm{CT} / \mathrm{NG}-$ Testsystem liegt eine Studie mit $4000 \mathrm{Va}$ ginalabstrichen vor [31]. Die vaginalen Abstriche identifizierten 92,9\% bzw. 98,5\% der Chlamydia-trachomatis- und N.-gonorrhoeae-Infektionen - deutlich mehr als aus anderen Probenmaterialien wie Endozervikalabstriche oder Urin, unabhängig davon, ob es ärztlich abgenommene Proben oder von den $\mathrm{Pa}$ tientinnen selbst entnommene Abstriche waren. Entscheidend für den Nachweis der Erreger ist, dass auch tatsächlich Zellmaterial am Abstrich bzw. im Urin enthalten sein muss, da es sonst zu falsch negativem Testausfall kommen kann.

\section{Extragenitale Gonorrhö}

Zum Ausschluss einer extragenitalen Gonorrhö sollten auch Anal- und ggf. Rachenabstriche entnommen werden, insbesondere bei Patienten, die Risikogruppen angehören (MSM, außerdem weib- liche Sexarbeiterinnen). Zu beachten ist, dass bestimmte NAT nicht für diese Materialien validiert sind, da es $\mathrm{zu}$ falsch positivem Reaktionsausfall wegen Kreuzreaktivität mit apathogenen NeisserienArten kommen kann.

\section{Mikroskopischer Nachweis der Gonokokken}

Im Grampräparat finden sich bei Gonorrhö gehäuft intraleukozytäre, gramnegative, semmelförmige Diplokokken. Bei Frauen gelingt der mikroskopische Nachweis der Gonokokken am häufigsten aus dem Zervikalsekret, bei Männern aus dem Urethralabstrich. Gramfärbung und Methylenblaupräparat besitzen insbesondere bei Frauen nur orientierenden Wert, da die Begleitflora die Abgrenzung erschwert.

\section{Kultureller Erregernachweis der Gonokokken}

Derkulturelle Erregernachweis auf Kochblut- oder Levinthal-Agar („Schokoladenagar") sowie dem Selektivmedium vom Typ Thayer-Martin sollte immer angestrebt werden, nicht zuletzt mit Blick auf die nur dadurch mögliche Empfindlichkeitstestung des Isolates [22]. Am effektivsten ist die Beimpfung der Nährmedien sofort nach der Materialabnahme vom Patienten. Idealerweise sollte der Abstrich innerhalb von $4 \mathrm{~h}$ im Labor sein, um möglichst schnell die GonokokkenKulturen ansetzen zu können. Die Bebrütung erfolgt für mindestens $48 \mathrm{~h}$ bei $36{ }^{\circ} \mathrm{C}$ im $\mathrm{CO}_{2}$-Brutschrank. Möglich ist auch die Schaffung eines mikroaerophilen Milieus $\left(\mathrm{CO}_{2}\right.$-Gehalt $\left.>5 \%\right)$ im sog. Kerzentopf (Entzünden eines Teelichtes im luftdichten Bebrütungsgefäß). Gonokokken bilden bei Wachstum in 2 Tagen feine, glatte, pigmentlose, grau-opake Kolonien von 0,6-1,5 mm Durchmesser (• Abb. 3).

\section{Identifizierung von Neisseria gonorrhoeae}

Die positive Oxidasereaktion gilt allenfalls als orientierender Bestätigungstest. Zusätzlich sollte N. gonorrhoeae von den kommensalen Neisserien-Arten bioche- misch durch das unterschiedliche Säurebildungsvermögen aus Glukose, Maltose, Fruktose, Saccharose und Laktose abgegrenzt werden. Dafür kann auf kommerziell verfügbare Testsysteme zurückgegriffen werden. Verwendet werden beispielsweise der manuell durchzuführende API-NH-Identifikations-Streifentest oder der Vitek ${ }^{\circledR}$ 2, ein kompaktes, automatisiertes ID/AST-System (beide von bioMérieux, Nürtingen).

\section{Molekularbiologischer Nachweis von Neisseria-gonorrhoeae-DNA}

Die Diagnosesicherung einer Gonorrhö erfolgt heute in erster Linie mittels molekularbiologischer Methoden. Die hohe diagnostische Empfindlichkeit des NATNachweises der N.-gonorrhoeae-DNA ist ein Grund dafür, dass der kulturelle Gonokokken-Nachweis mehr und mehr in den Hintergrund gerückt ist [15]. Das ist jedoch - wie oben ausgeführt - problematisch mit Blick auf die dann nicht mögliche In-vitro-Empfindlichkeitstestung der Isolate.

Folgende NAT-Testsysteme sind beispielsweise im Einsatz: Gen-Probe Aptima Combo 2 transcription-mediated amplification assay (TMA), BD (Becton Dickinson) ProbeTec ET amplified DNA strand displacement assay (SDA) und der klassische Roche Cobas Amplicor PCR (Polymerasekettenreaktion)-Test. Die Empfindlichkeit der Methoden ist sehr hoch. In einer Studie lag die diagnostische Sensitivität bei von Patientinnen selbst entnommenen Vaginalabstrichen für die PCR Roche und für TMA GenProbe bei jeweils $100 \%$, der SDA wies $80 \%$ auf [21].

Eine spanische Studie verglich den kulturellen Gonokokken-Nachweis mit einer Real-Time-PCR [27]. Von den 768 Proben waren 96,9\% konkordant. So waren 21 Proben PCR-positiv und Kultur-negativ, dagegen nur 3 Proben PCR-negativ und Kultur-positiv. Diagnostische Empfindlichkeit, Spezifität, positiver und negativer Vorhersagewert betrugen für die Kultur 86,2\%, 99,8\%, $99,2 \%$ und $96,7 \%$, die PCR war mit $98,7 \%, 100 \%, 100 \%$ und $99,7 \%$ überlegen. 
Problematisch ist der Nachweis einer pharyngealen Gonorrhö, hierbei versagt die kulturelle Anzucht des Erregers in $50 \%$ der Fälle, da diverse apathogene Neisseria-Stämme stören. Die Amplifikationstechniken sind deutlich sensitiver (PCR Roche 80,3\%, Gen-Probe TMA 83,6\% und BD ProbeTec 93,2 \%), jedoch nicht so empfindlich wie bei urethralen, zervikalen oder vaginalen Abstrichen [1].

\section{》) Die Amplifikationstechniken sind deutlich sensitiver als die Kultur}

Eine Therapieerfolgskontrolle mit NATNachweis sollte frühestens 14 Tage nach Beendigung der Therapie erfolgen. Wenn zu früh nachkontrolliert wird, sind falsch positive Ergebnisse möglich.

\section{Resistenzsituation}

Vor dem Hintergrund steigender Antibiotikaresistenzen hat die routinemäßige Empfindlichkeitstestung derN.-gonorrhoeae-Isolate für den Therapieerfolg $\mathrm{Be}$ deutung. Außerdem ist die Beurteilung der Epidemiologie der Antibiotikaresistenzen der Gonokokken möglich. Das gilt insbesondere dann, wenn, wie auch vom Erstautor dieses Artikels praktiziert, alle isolierten N.-gonorrhoeae-Stämme an das Konsiliarlabor für Gonokokken in Berlin (Prof. Dr. Peter K. Kohl, Vivantes Klinikum Berlin-Neukölln) gesendet werden. Gerade hat das Robert Koch-Institut mit dem Konsiliarlabor das Gonokokken Resistenz Netzwerk (GORENET) gegründet. Ziel des Projektes ist die Verbesserung der Datenlage zur Gonokokken-Resistenz in Deutschland, um einen besseren Überblick über die Resistenzlage $\mathrm{zu}$ bekommen und evidenzbasiert die Therapieempfehlungen anpassen $\mathrm{zu}$ können sowie Empfehlungen für zielgerichtete Präventionsmaßnahmen im Gesundheitswesen $\mathrm{zu}$ entwickeln.

\section{Resistenzsituation von Neisseria gonorrhoeae in Asien}

Aus Japan stammte der erste "Superbug“ N. gonorrhoeae, ein Stamm, der gegen nahezu alle verfügbaren Antibiotika re- sistent war, insbesondere natürlich gegen die aktuell zur Therapie empfohlenen Cephalosporine wie Ceftriaxon und Cefixim [24, 29]. Wiederholt schon hat die WHO ihre Sorge ausgedrückt, dass solche „high level“-resistenten Isolate von N. gonorrhoeae sich weltweit ausbreiten, sodass die Gonorrhö möglicherweise eine mit Antibiotika nicht mehr behandelbare Infektion werden könnte [30].

Exemplarisch für die Situation der N.gonorrhoeae-Resistenz in Asien stehen die aktuellen Zahlen aus Nordindien. Dort stieg die Rate der Penicillinase bildenden Stämme von $8 \%$ in den Jahren 1995-1996 auf $20 \%$ von 2004-2005 und zuletzt $88 \%$ (2011-2013) [2]. Die Raten der Chinolon-resistenten Stämme stiegen von 12\% (1995-1996) auf 98,3\% (2004-2005), danach gab es einen geringen Abfall auf 84\% (2011-2013). Die hohen Resistenzraten in Indien unterstreichen, dass Ciprofloxacin für die kalkulierte Behandlung der Gonorrhö nicht mehr infrage kommt. Im Januar 2013 wurden in Nordindien die ersten Gonokokken-Isolate mit verminderter Empfindlichkeit gegenüber Cephalosporinen der 3. Generation (Ceftriaxon, Cefixim und Cefpodoxim) gefunden (MHK [minimale Hemmkonzentration] -Wert für Ceftriaxon $=0,19 \mu \mathrm{g} / \mathrm{ml}$ ).

\section{In-vitro-Empfindlichkeit von Neisseria gonorrhoeae in Deutschland}

Im Gegensatz zu Treponema pallidum besitzt N. gonorrhoeae außerordentlich vielfältige Mechanismen der Anpassung und Resistenzentwicklung (Resistenz durch chromosomale Mutationen, $\beta$-Laktamase-Plasmide u.a.). In Deutschland hat die Paul-Ehrlich-Gesellschaft für Chemotherapie (PEG) eine Studie zur Antibiotikaempfindlichkeit von N. gonorrhoeae durchgeführt. Im Zeitraum von Oktober 2010 bis Dezember 2011 wurden 213 Gonokokken-Isolate aus 23 Laboren untersucht. Gegenüber Penicillin waren $80 \%$ der Isolate in vitro nicht empfindlich. Für Ciprofloxacin fand sich mit $74 \%$ die höchste Resistenzrate, es folgte Doxycyclin mit $41 \%$. Eine Azithromycin-Resistenzlag bei $6 \%$ der Isolate vor. Dagegen waren alle Stämme empfindlich gegen- über Spectinomycin, Cefixim und Ceftriaxon [14]. Im Rahmen des European Surveillance (European gonococcal antimicrobial surveillance programme oder Euro-GASP) fanden sich für Deutschland im Jahr 2013 folgende Resistenzraten für N. gonorrhoeae: Cefixim 12,9\%, Ciprofloxacin 63,4\% und Azithromycin $4 \%$ [9]. Insbesondere der hohe Prozentsatz Cefixim-resistenter Stämme sollte Anlass sein, die orale Cefixim-Gabe in Deutschland sehr kritisch zu sehen.

\section{In-vitro-Empfindlichkeit und} Multidrug-Resistenz von Neisseria gonorrhoeae in Europa

In den europäischen Ländern ist eine Zunahme der sexuell übertragenen Erkrankungen zu verzeichnen, parallel dazu nehmen Antibiotikaresistenzen von Gonokokken zu. Die seit mindestens 10 Jahren zu beobachtende Entwicklung der Erhöhung der In-vitro-Resistenzraten von N. gonorrhoeae in Europa und Deutschland setzt sich weiter fort [8]. In Österreich hat man von 2010 bis 2014 bei 3584 Gonokokken-Isolaten die MHK mittels Agardilution und den Breakpoint mittels $\mathrm{E}^{\circledR}$-Test bestimmt [28]. In der Untersuchungsperiode hatte sich die Anzahl der Gonokokken-Infektionen in Wien verachtfacht. Die Resistenzraten gegenüber Chinolonen, Penicillin und Doxycyclin hatten sich deutlich erhöht; 21 Isolate waren Cefixim-resistent. Die Azithromycin-Resistenz stieg von 0,9\% (2013) auf 3,2\% (2014) an. Ceftriaxonresistente Stämme wurden nicht gefunden.

\section{) In Europa nehmen die Antibiotikaresistenzen von Gonokokken zu}

Im Jahr 2013 wurden insgesamt 1994 Isolate aus 21 europäischen Ländern getestet [9]: 52,9\% waren Ciprofloxacin-resistent (2012: 50,1\%) [8]. Azithromycin-Resistenz fand sich bei 5,4\% der Isolate. Bemerkenswert ist, dass 93 (4,7\%) der untersuchten 1994 Stämme gegenüber Cefixim resistent waren. Cefixim-resistente Isolate fanden sich in 13 der beteiligten 21 Länder. Es waren vorzugsweise Frau- 
en und männliche Heterosexuelle, die ein signifikant höheres Risiko für eine Infektion durch Cefixim-resistente N. gonorrhoeae aufwiesen. Sieben Stämme (0,4 \%) waren Ceftriaxon- und gleichzeitig Cefixim-resistent. Alle 93 Cefixim-resistente Isolate waren auch Ciprofloxacin-resistent, 16 (17,2 \%) Stämme waren zusätzlich Azithromycin-resistent.

\section{Therapie von Neisseria- gonorrhoeae-Infektionen}

Das Spektrum der Antibiotika zur Therapie ist aktuell aufgrund der stark angestiegenen Resistenzraten v. a. bei Fluorchinolonen (z. B. Ciprofloxacin) stark eingeschränkt [18, 19, 26, 29]. Mittel der Wahl für die antibiotische Behandlung der unkomplizierten Gonorrhö ist Ceftriaxon i.m., alternativ i.v., immer in Kombination mit Azithromycin als sog. duale Therapie. Wenn eine i. m.-Verabreichung kontraindiziert und eine i. v.Verabreichung nicht möglich ist, kann statt Ceftriaxon Cefixim (p. o.) verwendet werden $[12,17]$. Die duale Therapie mit gleichzeitiger Verabreichung von Ceftriaxon (bzw. Cefixim) und Azithromycin hat den Vorteil, dass eine möglicherweise gleichzeitig vorliegende ChlamydienInfektion mit behandelt wird [3]. Durch den doppelten Ansatz gegenüber N. gonorrhoeae bietet sich ein weiterer Angriffspunkt zur Therapie der Gonorrhö an, und es wird der Resistenzentwicklung des Cephalosporins vorgebeugt [17]. In vitro gibt es Hinweise auf eine wohl synergistische oder auch additive Wirkung von Cephalosporinen und Azithromycin [3].

\section{》) Eine duale Therapie mit Ceftriaxon (bzw. Cefixim) und Azithromycin wird empfohlen}

Die Standardtherapie der Gonorrhö erfolgt entsprechend dem aktuellen Leitfaden STI-Therapie, herausgegeben von der Deutschen STI-Gesellschaft (DSTIG) [10]. Der STI-Leitfaden wiederum basiert auf der AWMF (Arbeitsgemeinschaft der Wissenschaftlichen Medizinischen Fachgesellschaften)-Leitlinie 059004 „Gonorrhoe bei Erwachsenen und
Adoleszenten“ [3]. Mittel der Wahl ist Ceftriaxon $1,0 \mathrm{~g}$ i.m. oder i.v. einmalig zusammen mit Azithromycin 1,5 g p.o. einmalig [10], bei Kontraindikation für i.m.-Injektion und wenn i.v. nicht möglich: Cefixim $800 \mathrm{mg}$ p.o. plus Azithromycin 1,5 g p.o. jeweils als Einmaldosis. Nur bei kulturell nachgewiesener Empfindlichkeit sollte eine Monotherapie mit entweder Cefuroxim $400 \mathrm{mg}$, Azithromycin 1,5 g oder Ciprofloxacin $500 \mathrm{mg}$ jeweils p. o. erfolgen. Eine Partnertherapie sollte angestrebt werden $[3,10]$.

\section{Behandlung einer kombinierten Infektion durch Neisseria gonorrhoeae und Chlamydia trachomatis}

Bei gleichzeitiger Gonorrhö und Chlamydien-Infektion kommt Ceftriaxon 1,0 g i. m. oder i. v. (einmalig) zusammen mit Azithromycin 1,5g p.o. (einmalig) zur Anwendung [4]. Auch hier gilt, dass bei Kontraindikation für i. m.-Injektion und wenn i.v. nicht möglich Cefixim $800 \mathrm{mg}$ p.o. plus Azithromycin 1,5 g p. o. jeweils als Einmaldosis verabreicht werden sollten $[3,10]$.

\section{Disseminierte Neisseria- gonorrhoeae-Infektionen}

Komplizierte und disseminierte N.-gonorrhoeae-Infektionen sollten immer stationär behandelt werden. Laut Leitfaden „STI-Therapie“ wird empfohlen, mit Ceftriaxon $1 \mathrm{~g}$ i. v. alle $24 \mathrm{~h}$ für mindestens 7 Tage oder länger zu behandeln [10]. Bei der akuten Beckenentzündung ist zu berücksichtigen, dass häufig Mischinfektionen mit Chlamydien und Anaerobiern (Bacteroides spp., Peptostreptococcus spp., Peptococcus spp.) und Enterobacteriaceae, außerdem auch mit Mycoplasma hominis vorliegen können. Deshalb kann eine Kombination des Cephalosporins mit anderen Antibiotika, z. B. mit Doxycyclin, oder die Gabe von Clindamycin plus Gentamicin erforderlich sein.
Therapie der Gonorrhö in der Schwangerschaft

In der Schwangerschaft ist Ceftriaxon $1 \mathrm{~g}$ i. v. (i. m.) als Einmaldosis die Therapie der Wahl.

\section{Credé-Prophylaxe}

Die Credé-Prophylaxe dient der Vorbeugung einer Gonoblennorrhö des Neugeborenen bei einer N.-gonorrhoeae-positiven Mutter, eine Konstellation, die heute in Deutschland selten zu erwarten ist. Das klassische Vorgehen besteht darin, einige Tropfen $1 \%$ iger Silbernitratlösung $\left(\mathrm{AgNO}_{3}\right)$ in den Konjunktivalsack des neugeborenen Säuglings einzubringen. Diese Methodik ist in Deutschland seit 1986 nicht mehr gesetzlich vorgeschrieben. Aufgrund der gründlichen vorgeburtlichen Untersuchungen und des geringen Anteils Gonokokken-infizierter Schwangeren wird heute oft ganz auf die Prophylaxe verzichtet. Dagegen steht, dass das Bundesgesundheitsamt 1992 darauf hingewiesen hat, dass neben der Gonorrhö auch andere Formen der Konjunktivitis von Relevanz sind, dass die Prophylaxe „standard of care“ darstellt und dass weltweit nicht ein einziger Fall bleibender Nebenwirkungen nach adäquater Anwendung von $1 \%$ Silbernitratlösung beobachtet wurde. Deshalb werden laut STI-Leitfaden zur Prophylaxe einer Gonoblennorrhö alternativ entweder $1 \%$ Silbernitrat oder antibiotikahaltige Augentropfen (Erythromycin/ Tetracyclin) oder PVP-Jod-Lösungen (Ophthalmikum) empfohlen [10].

\section{Behandlung des Sexualpartners}

Laut STI-Leitfaden [10] wird die Partnertherapie bei Gonorrhö empfohlen. Aus forensischen Gründen sollte die Partnerin/der Partner jedoch nach Aufklärung einer solchen Behandlung zuvor zugestimmt haben. Auf die alternative Möglichkeit der (wiederholten) Kontrolluntersuchungen sollte hingewiesen werden.

\section{Fazit für die Praxis}

\section{- Die Gonorrhö wird nahezu aus- schließlich sexuell übertragen und betrifft vornehmlich die Schleimhaut}




\section{des Urogenitaltraktes, möglich sind jedoch auch extragenitale Lokalisa- tionen. \\ - Besorgniserregend ist die Zunahme der Resistenz von Neisseria gonor- rhoeae gegenüber Penicillin, Doxy- cyclin, Ciprofloxacin und neuerdings auch gegenüber Azithromycin und Drittgenerationscephalosporinen. \\ - Die Standardtherapie der Gonorrhö erfolgt heute mit Ceftriaxon (i. v. oder i. m.) zusammen mit Azithromycin p. o., beides als Einmalgabe.}

\section{Korrespondenzadresse}

Prof. Dr. P. Nenoff

Labor für medizinische Mikrobiologie, Partnerschaft Prof. Dr. med. Pietro Nenoff \& Dr. med. Constanze Krüger

Mölbiser Hauptstr. 8, 04571 Rötha/OT Mölbis, Deutschland

nenoff@mykologie-experten.de

\section{Einhaltung ethischer Richtlinien}

Interessenkonflikt. P. Nenoff, A. Manos, I. Ehrhard, C. Krüger, U. Paasch, P. Helmbold und W. Handrick geben an, dass kein Interessenkonflikt besteht.

Dieser Beitrag beinhaltet keine von den Autoren durchgeführten Studien an Menschen oder Tieren.

\section{Literatur}

1. Bachmann LH, Johnson RE, Cheng H et al (2009) Nucleic acid amplification tests for diagnosis of Neisseria gonorrhoeae oropharyngeal infections. JClin Microbiol 47:902-907

2. Bharara T, Bhalla P, Rawat D et al (2015) Rising trend of antimicrobial resistance among Neisseria gonorrhoeae isolates and the emergence of $\mathrm{N}$. gonorrhoeae isolate with decreased susceptibility to ceftriaxone. Indian J Med Microbiol 33:39-42

3. Bremer V, Brockmeyer NH, Buder $\mathrm{S}$ et al (2013) Gonorrhoe bei Erwachsenen und Adoleszenten. AWMF-Leitlinie 08/2013. http:// www.awmf.org/uploads/tx_szleitlinien/059004l_S25_Gonorrhoe_bei_Erwachsenen_ Adoleszenten_2014-verlaengert_01.pdf. Zugegriffen:23.11.2016

4. Campos-Outcalt $D$ (2011) CDC update on gonorrhea: expand treatment to limit resistance. J Fam Pract 60:736-739

5. Centers for Disease Control and Prevention (2014) Gonorrhea - CDC Fact Sheet (Detailed Version). Atlanta, GA: U.S. Department of Health and Human Services, Centers for Disease Control and Prevention. http://www.cdc.gov/std/Gonorrhea/ STDFact-gonorrhea-detailed.htm. Zugegriffen: 4.4.2014

6. CDC (2016) CDC's yearly Sexually Transmitted Disease Surveillance Report 2015. Division of STD
Prevention. https://www.cdc.gov/std/stats15/. Zugegriffen:26.10.2016

7. Chow EP, Cornelisse VJ, Read TR et al (2016) Saliva use as a lubricant for anal sex is a risk factor for rectal gonorrhoea among men who have sex with men, a new public health message: a cross-sectional survey. Sex Transm Infect (Epub ahead of print)

8. Cole MJ, Spiteri G, Chisholm SA et al (2014) Emerging cephalosporin and multidrug-resistant gonorrhoea in Europe. Euro Surveill 19:20955

9. Cole MJ, Spiteri G, Jacobsson S et al (2015) Is the tide turning again for cephalosporin resistance in Neisseria gonorrhoeae in Europe? Results from the 2013 European surveillance. BMC Infect Dis 15:321

10. Deutsche STI-Gesellschaft (DSTIG) (2015) Leitfaden STI-Therapie, 2. Aufl. Deutsche STI-Gesellschaft e.V. Gesellschaft zur Förderung der Sexuellen Gesundheit, Bochum. http://dstig. de/images/DSTIG-Flyer/Leitfaden/sti-leitfaden_ version\%202.1_web.pdf.Zugegriffen:23.11.2016

11. European Centre for Disease Prevention and Control (2016) Annual epidemiological report 2016 - Gonorrhoea. http://ecdc.europa.eu/ en/healthtopics/Gonorrhoeae/Pages/Annualepidemiological-report-2016.aspx. Zugegriffen: 22.11.2016

12. RKI (2013) Gonorrhö (Tripper). RKI-Ratgeber für Ärzte. https://www.rki.de/DE/Content/Infekt/ EpidBull/Merkblaetter/Ratgeber Gonorrhoe.html;jsessionid=EE300AE95D 25055EE6A74DAEA25AB68C.2_cid363\# doc3763050bodyText11.Zugegriffen: 27.10.2016

13. Haustein UF (1990) Sexuell übertragbare Krankheiten, 1. Aufl. Gustav Fischer, Jena

14. Horn NN, Kresken M, Körber-Irrgang B et al (2014) Antimicrobial susceptibility and molecular epidemiology of Neisseria gonorrhoeae in Germany. Int J Med Microbiol 304:586-591

15. Horner PJ, Blee K, Falk L, van der Meijden W, Moi $H$ (2016) 2016 European guideline on the management of non-gonococcal urethritis. Int J STD AIDS 27:928-937

16. Hublikar S, Maher WE, Bazan JA (2014) Disseminated gonococcal infection and eculizumab - a "high risk" connection? Sex Transm Dis 41:747-748

17. Kidd S, Workowski KA (2015) Management of gonorrhea in adolescents and adults in the United States. Clin Infect Dis 61(S8):S785-S801

18. Kirkcaldy RD, Hook EW 3rd, Soge $O 0$ et al (2015) Trends in Neisseria gonorrhoeae susceptibility to cephalosporins in the United States, 2006-2014. JAm Med Assoc 314:1869-1871

19. Lancaster JW, Mahoney MV, Mandal S, Lawrence KR (2015) Update on treatment options for gonococcal infections. Pharmacotherapy 35:856-868

20. Le Roy C, Hénin N, Pereyre S, Bébéar C (2016) Fluoroquinolone-resistant mycoplasma genitalium, Southwestern France. Emerg Infect Dis 22:1677-1679

21. MasekBJ, Arora N, Quinn Netal (2009) Performance of three nucleic acid amplification tests for detection of Chlamydia trachomatis and Neisseria gonorrhoeae by use of self-collected vaginal swabs obtained via an Internet-based screening program. Clin Microbiol 47:1663-1667

22. Nenoff P, Hillert R, Handrick W et al (2010) Genitale Neisseria gonorrhoeae-Infektionen - aktuelle Aspekte zur Epidemiologie, Labordiagnostik, Resistenzsituation und Therapie. Gyn Prakt Gynäkol 15:182-196

23. Nenoff $P$, Manos A, Krüger C, Handrick W (2016) Infektionen durch Chlamydien, Gonokokken, Treponema pallidum, Mykoplasmen, Ureaplasma und Trichomonaden. Aktuelle Aspekte der
Epidemiologie, Labordiagnostik und Therapie. Gyne 37:25-30

24. Ohnishi M, Golparian D, Shimuta K et al (2011) Is Neisseria gonorrhoeae initiating a future era of untreatable gonorrhea?: detailed characterization of the first strain with high-level resistance to ceftriaxone. Antimicrob Agents Chemother 55:3538-3545

25. Papp JR, Schachter J, Gaydos CA, Van Der Pol $B$ (2014) Recommendations for the laboratorybased detection of Chlamydia trachomatis and Neisseria gonorrhoeae - 2014. Recommendations and Reports. MMWR63:1-19

26. Pogany L, Romanowski B, Robinson J et al (2015) Management of gonococcal infection among adults and youth. Can Fam Physician 61:869-873

27. Rumyantseva T, Golparian D, Nilsson CS et al (2015) Evaluation of the new AmpliSens multiplex real-time PCR assay for simultaneous detection of Neisseria gonorrhoeae, Chlamydia trachomatis, Mycoplasma genitalium, and Trichomonas vaginalis. APMIS 123:879-886

28. Stary A, Heller-Vitouch C, Binder M et al (2015) Die Gonokokkeninfektion in Österreich: eine Langzeitbeobachtung der Prävalenz und Resistenzentwicklung von 1999 bis 2014. J Dtsch Dermatol Ges 13:1136-1146

29. Unemo M (2015) Current and future antimicrobial treatment of gonorrhoea - the rapidly evolving Neisseria gonorrhoeae continues to challenge. BMCInfect Dis 15:364

30. Unemo M, Nicholas RA (2012) Emergence of multidrug-resistant, extensively drug-resistant and untreatable gonorrhea. Future Microbiol 7:1401-1422

31. Van Der Pol B, Taylor SN, Liesenfeld $O$ et al (2013) Vaginal swabs are the optimal specimen for detection of genital Chlamydia trachomatis or Neisseria gonorrhoeae using the Cobas 4800 CT/NG test. Sex Transm Dis 40:247-250

32. Verordnung des Sächsischen Staatsministeriums für Soziales und Verbraucherschutz über die Erweiterung der Meldepflicht für übertragbare Krankheiten und Krankheitserreger nach dem Infektionsschutzgesetz (IfSGMeldeVO) vom 3. Jun 2002, rechtsbereinigt mitStand vom 16. Dezember 2012, http://www.revosax.sachsen.de/vorschrift/ 1307-IfSGMeldeVO.Zugegriffen:23.11.2016 